\title{
Anisotropic behavior of quantum transport in graphene superlattices \\ Coexistence of ballistic conduction with Anderson insulating regime
}

Pedersen, Jesper Goor; Cummings, Aron W.; Roche, Stephan

Published in:

Physical Review B

Link to article, DOI:

10.1103/PhysRevB.89.165401

Publication date:

2014

Document Version

Publisher's PDF, also known as Version of record

Link back to DTU Orbit

Citation (APA):

Pedersen, J. G., Cummings, A. W., \& Roche, S. (2014). Anisotropic behavior of quantum transport in graphene superlattices: Coexistence of ballistic conduction with Anderson insulating regime. Physical Review B, 89(16), 165401. https://doi.org/10.1103/PhysRevB.89.165401

\section{General rights}

Copyright and moral rights for the publications made accessible in the public portal are retained by the authors and/or other copyright owners and it is a condition of accessing publications that users recognise and abide by the legal requirements associated with these rights.

- Users may download and print one copy of any publication from the public portal for the purpose of private study or research.

- You may not further distribute the material or use it for any profit-making activity or commercial gain

- You may freely distribute the URL identifying the publication in the public portal 


\title{
Anisotropic behavior of quantum transport in graphene superlattices: Coexistence of ballistic conduction with Anderson insulating regime
}

\author{
Jesper Goor Pedersen, ${ }^{1,2}$ Aron W. Cummings, ${ }^{2}$ and Stephan Roche ${ }^{2,3}$ \\ ${ }^{1}$ DTU Nanotech-Department of Micro- and Nanotechnology, Technical University of Denmark, DK-2800 Kgs. Lyngby, Denmark \\ ${ }^{2}$ Institut Català de Nanociència i Nanotecnologia (ICN2), Campus de la UAB, Edifici ICN2, 08193 Bellaterra, Barcelona, Spain \\ ${ }^{3}$ ICREA - Institucio Catalana de Recerca i Estudis Avancats, 08010 Barcelona, Spain
}

(Received 30 October 2013; published 1 April 2014)

\begin{abstract}
We report on the possibility to generate highly anisotropic quantum conductivity in disordered graphene-based superlattices. Our quantum simulations, based on an efficient real-space implementation of the Kubo-Greenwood formula, show that in disordered graphene superlattices the strength of multiple scattering phenomena can strongly depend on the transport measurement geometry. This eventually yields the coexistence of a ballistic waveguide and a highly resistive channel (Anderson insulator) in the same two-dimensional platform, evidenced by a $\sigma_{y y} / \sigma_{x x}$ ratio varying over several orders of magnitude, and suggesting the possibility of building graphene electronic circuits based on the unique properties of chiral massless Dirac fermions in graphene.
\end{abstract}

DOI: 10.1103/PhysRevB.89.165401

PACS number(s): 73.23.-b, 72.15.Rn, 73.21.Cd

Graphene has received significant interest for its unusual transport properties, including phenomena such as Klein tunneling [1] and weak antilocalization [2]. These phenomena preclude the formation of an insulating state at low levels of disorder, in contrast to traditional 2D metallic systems, which are predicted to exhibit insulating states for arbitrarily small disorder strengths $[3,4]$. This unique behavior makes graphene a promising material for a wide range of nextgeneration technological applications [5]. One challenging research objective is to maximize the transport anisotropy of graphene, such that it remains a good conductor along one direction while being much more resistive along the other. Such control over the transport properties could be used to direct charge flow and build electronic circuits or waveguides [6], optical circuits [7], or communications devices [8]. For most scattering sources, the transport properties in disordered graphene remain isotropic. More complex forms of disorder, such as structural deformations produced by interaction with a substrate $[9,10]$ or chemical doping [11], produce only moderate transport anisotropies.

A way around this problem involves the creation of graphene superlattices, which are formed from a periodic modulation of the electrical, mechanical, or chemical properties of pristine graphene. It has been theoretically predicted that a strongly anisotropic Fermi velocity can be induced by patterned periodic modifications of the graphene surface [12]. Based on this work, the construction of novel types of graphene electronic devices and circuits from structurally-perfect engineered superlattices was foreseen, attracting significant attention [13-15]. To date however, the inherent disorder introduced through conventional lithography techniques and top-down patterning approaches has impeded the realization of graphene-based superlattices, whose novel transport and device characteristics depend on band-structure engineering, and are thus highly sensitive to disorder [16].

Here, we wish to advocate a different approach to nanostructuring graphene, where instead of considering disorder an inevitable drawback, it serves as beneficial for tuning the properties of graphene. While structural disorder inherent to any fabricated graphene-based superlattice jeopardizes the control of band-structure features, we demonstrate that disorder enables the coexistence of transport regimes which are generally incompatible in conventional conducting materials. Using efficient numerical methodologies, the scaling properties of quantum transport through disordered graphenebased superlattices are demonstrated to become exceedingly anisotropic, resulting in the coexistence of a ballistic transport channel in one direction and a highly resistive channel in the other. We note that similar ideas have been proposed within the field of photonic crystals [17].

We consider a disordered, rectangular lattice of perforations in an otherwise pristine graphene sheet. A given superlattice is referred to via parameters $\left\{N_{x}, N_{y}\right\}$, with $N_{\mu}$ denoting the average number of hexagons between the center of the holes along each direction. The lattice constants of the pristine superlattice are $A_{x}=\sqrt{3} N_{x} a$ and $A_{y}=N_{y} a$, respectively, with $a=0.246 \mathrm{~nm}$ the graphene lattice constant. The average size of the antidots is defined via the radius $R$, which we fix at $R=3 a$. We consider superlattices for which $N_{x} / N_{y}>1$ and refer to the $y$ and $x$ directions as the easy and the hard directions, respectively. Disorder is included via parameters $\delta_{x}, \delta_{y}, \delta_{R}, p_{\text {rem }}$, and $N_{\text {rem. }}$. Here, $\delta_{x}, \delta_{y}$, and $\delta_{R}$ denote the widths of random fluctuations in the centers and radii of holes, respectively. Edge disorder along the holes is added via an iterative process repeated $N_{\text {rem }}$ times, in which edge atoms are removed with probability $p_{\text {rem. }}$. We remove any dangling bonds in the geometry, i.e., all carbon atoms have at least two nearest neighbors. While structural relaxation is not included, the absence of dangling bonds leads us to expect only a minor influence of potential geometrical relaxation [18]. Throughout this paper we take $\delta_{x}=\delta_{y}=2 a, \delta_{R}=0.5 a$, $p_{\text {rem }}=5 \%$, and $N_{\text {rem }}=2$. An example of a disordered $\{16,9\}$ superlattice is illustrated in Fig. 1. Note that the figure only shows a small, representative domain of the full structure used in the calculations, which contains several millions of atoms. We stress that while we only show results for $N_{x}>N_{y}$, we have confirmed that the results do not depend qualitatively on the orientation of the superlattice with respect to the graphene sheet.

We employ a nearest-neighbor tight-binding model with hopping element $t=-3 \mathrm{eV}$ and set onsite energies to zero. Transport properties are determined numerically via 


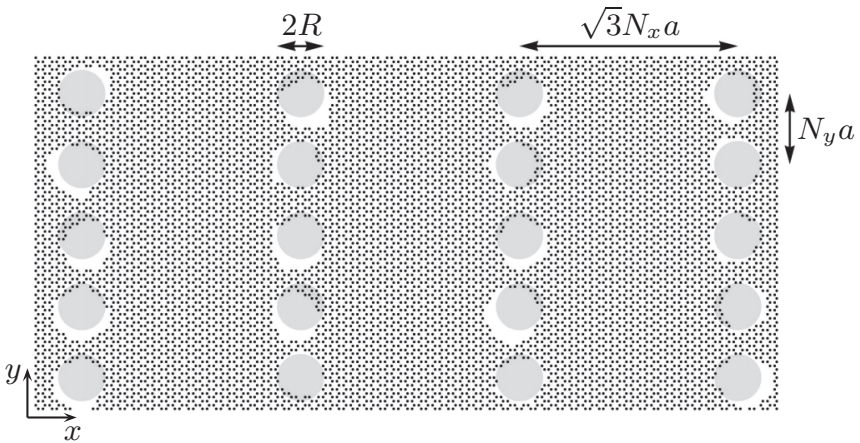

FIG. 1. Geometry of a representative domain of a disordered $\{16,9\}$ superlattice. Carbon atoms are indicated with black points. The gray shading indicates the position of holes in the pristine geometry.

an order- $N$ real-space Kubo approach [19-22]. The timeand energy-dependent diffusion coefficient is calculated via $D_{x}(E, t)=\Delta X(E, t)^{2} / t$, with the mean quadratic displacement $\Delta X(E, t)^{2}=\operatorname{Tr}\left[\delta(E-\hat{H})|\hat{X}(t)-\hat{X}(0)|^{2}\right] / \rho(E)$, where $\rho(E)=\operatorname{Tr}[\delta(E-\hat{H})]$ represents the density of states. Traces are approximated via initial random phase states and the Lanczos method [23], while the time evolution operator is expanded in Chebyshev polynomials. We use 1500 iterations in the Lanczos method, with broadening included via an imaginary part $\eta=0.04 \mathrm{eV}$ of the energy, while the number of Chebyshev polynomials is determined by requiring unitarity of the time evolution operator (within an error of $<10^{-6}$ ). The Kubo conductivity, including quantum interference effects, can be calculated from the diffusion coefficients as $\sigma_{x x}(E, t)=e^{2} \rho(E) D_{x}(e, t) / 2$, while the semiclassical conductivity reads $\sigma_{x x}^{\mathrm{SC}}(E, t)=e^{2} \rho(E) \max _{t} D_{x}(E, t) / 2$. Transport properties along the $y$ direction are calculated similarly.

In Fig. 2(a), solid lines illustrate the density of states (DOS) for a pristine $\{16,9\}$ superlattice. Note the quasi-onedimensional nature of the DOS with signatures of van Hove singularities, consistent with a picture of the superlattice as
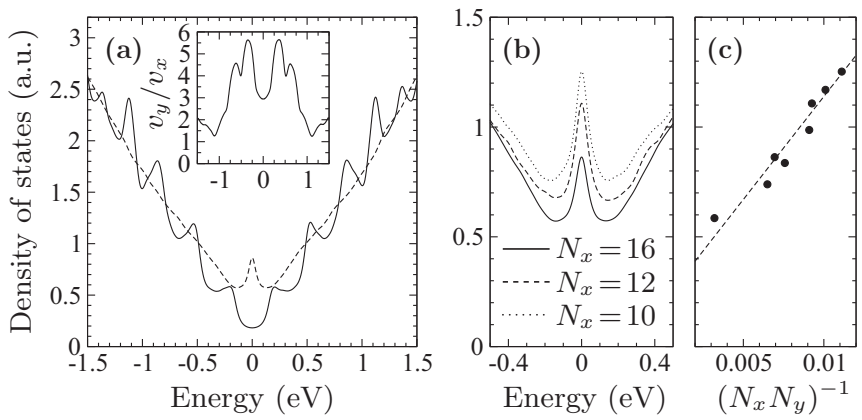

FIG. 2. (a) Density of states of a pristine (solid line) and a disordered (dashed line) $\{16,9\}$ superlattice. Note the absence of any signatures of van Hove singularities for the disordered structure. The inset shows the ratio of group velocities $v_{y} / v_{x}$ of the pristine superlattice. (b) Density of states near the Dirac point for three different disordered superlattices $\left\{N_{x}, 9\right\}$. (c) Density of states at the Dirac point for several disordered superlattices, illustrating the approximately linear dependence of the Dirac point DOS on the hole density. an array of weakly coupled nanoribbons aligned along the easy direction. The large aspect ratio of the superlattice unit cell results in a substantial anisotropy of the group velocity $v_{y} / v_{x}$, as illustrated in the inset of Fig. 2(a). Note that for larger energies (not shown), such anisotropy is much less pronounced, as expected from the suppression of band structure effects for energies where the electron wavelength becomes much smaller than the hole spacing. While an anisotropic Fermi velocity should affect transport properties, any effect relying critically on periodicity is bound to be quenched significantly in experimental realizations due to the presence of disorder.

We here consider this issue by focusing on lattices displaying significant structural imperfections, inspired by recent experimental fabrications of graphene superlattices [24]. In stark contrast to the pristine case, the DOS of such disordered structures [dashed line in Fig. 2(a)] becomes largely featureless, except for a peak at the Dirac point. The disorder is thus sufficiently strong to wash out the characteristics of the band structure associated with the superlattice. Figure 2(b) shows a zoom of the zero-energy DOS peak. While the DOS resembles that of bulk graphene with short-range impurities [25], the source of the zero-energy peak is different in our case. We attribute the zero-energy peak to states localized predominantly on the hole edges, emerging due to local sublattice imbalances $[18,26]$. Consistent with this picture, we illustrate in Fig. 2(c) the proportionality of the zero-energy DOS with the density of holes, quantified here via the dimensionless quantity $1 /\left(N_{x} N_{y}\right)$.

As discussed above, the disorder considered is strong enough to quench any effects emerging due to the periodicity of the superlattice (such as anisotropic group velocities). Instead, the scattering sources are determined by the randomness in the positions, radii, and internal geometries of the holes. Despite the largely featureless density of states, this results in drastically different transport properties along the two directions, as illustrated in Fig. 3, showing the diffusion coefficients $D_{\mu}(E, t)$ for different energies within $1 \mathrm{eV}$ of the Dirac point. Along the easy direction, the time dependence of the diffusion coefficients exhibits (within our accessible timescale of $20 \mathrm{ps}$ ) a quasiballistic behavior since $D_{\mu}$ does not saturate. In sharp contrast, along the hard axis $D_{\mu}(E, t)$ manifests a weak increase at short times before reaching its maximum value and then strongly decays in time, as a result of quantum interferences and localization phenomena [27]. We note that the total elapsed time of 20 ps considered in our calculations corresponds to sample dimensions on the order of 800-1000 nm along the easy direction and 100-180 nm along the hard direction [using $L_{\mu}(E)=2 \sqrt{t_{\max } D_{\mu}\left(E, t_{\max }\right)}$ ]. These results suggest that not only will conductivities along the two directions be of different orders of magnitude, but the temperature dependence should also indicate different transport regimes. In particular, for longer elapsed times (corresponding to wave-packet exploration of larger samples), a metallic behavior for $\sigma_{y y}(T)$ should be measured along one direction, while an insulating behavior for $\sigma_{x x}(T)$ should develop along the other.

In Fig. 3(c) we show the maximum value of the diffusion coefficient within the simulated time span. We attribute the sharp dip near zero energy to the localized zero-energy modes 


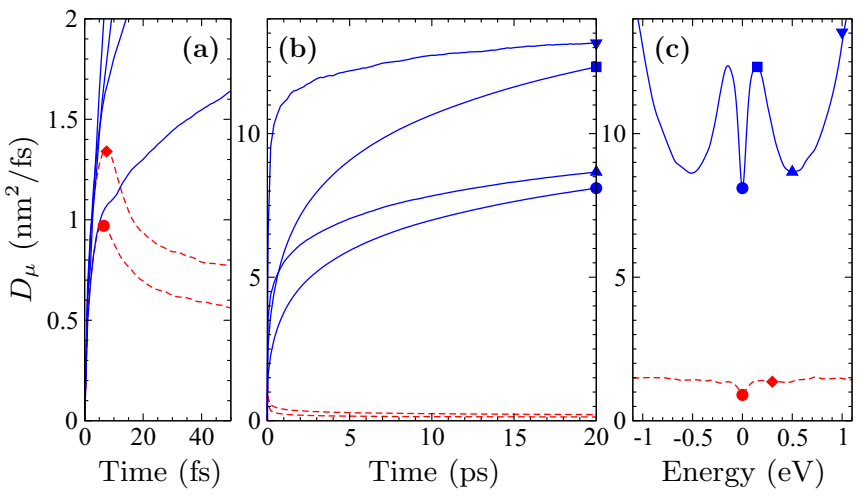

FIG. 3. (Color online) Time- and energy-dependent diffusion coefficients $D_{\mu}$ for a disordered $\{16,9\}$ superlattice. Solid blue (red dashed) lines show the diffusion coefficient along the easy (hard) direction. (a) Short-time behavior of $D_{\mu}$, illustrating strong localization along the hard direction. (b) Long-time behavior of $D_{\mu}$, illustrating quasi-ballistic transport along the easy direction. (c) Maximum diffusion coefficients within the simulation time span, shown versus energy. The symbols indicate the energies to which results in panels a and b correspond.

discussed above. As these states are predominantly localized around hole edges, they are naturally more sensitive to multiple scattering phenomena. Indeed, increasing the edge disorder further deepens the zero-energy feature, while features outside the energy range of the DOS peak are comparably much less affected. Furthermore, we find that the peaks in the mean free path on either side of the Dirac point coincide with the minimum of the DOS at the corresponding energies (see Fig. 4).

We extend our analysis by superimposing a random distribution of long-range impurities over the disordered superlattice potential. This enforces a saturation of the diffusion coefficients along both directions, giving access to mean free paths. We consider the addition of $N_{\mathrm{I}}$ long-range impurities, included in the onsite energies $V_{n}=\langle n|H| n\rangle$ as $V_{n}=$ $\sum_{i}^{N_{\mathrm{I}}} \epsilon_{i} \exp \left[-\left|\mathbf{r}_{n}-\mathbf{r}_{i}\right|^{2} /\left(2 \xi^{2}\right)\right]$, where $\xi$ sets the effective range, while the $\epsilon_{i} \in[-W / 2, W / 2]$ are randomly distributed [28-30]. We take usual parameters $W=|t|$ and $\xi=\sqrt{3} a$,
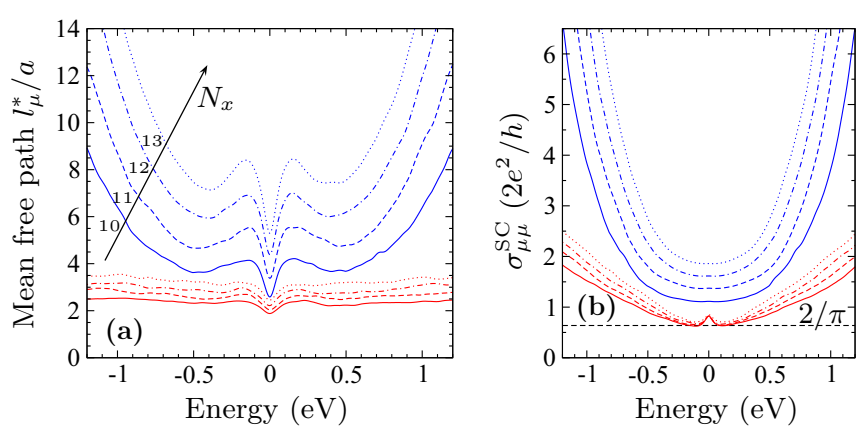

FIG. 4. (Color online) (a) Directional mean free paths $l_{e, \mu}^{*}$, in units of the graphene lattice constant, for disordered superlattices $\left\{N_{x}, 9\right\}$ with long-range impurities. Red lines show results for the "hard" direction, $l_{e, x}^{*}$, while blue lines indicate results for the "easy" direction, $l_{e, y}^{*}$. (b) Corresponding semiclassical conductivities, in units of $2 e^{2} / h$, along each direction. and consider a very low impurity concentration of $0.02 \%$, sufficient to give rise to a diffusive regime but low enough so that scattering remains dominated by the superlattice-driven random potential. The elastic mean free path is calculated from the maximum of the diffusion coefficient, $l_{e}(E)=$ $D_{\max }(E) /\left(2 v_{F}\right)$, with $v_{F}$ the Fermi velocity and $D_{\max }(E)$ the maximum of the average diffusion coefficient, $D(E, t)=$ $\left[D_{x}(E, t)+D_{y}(E, t)\right] / 2$. To study the transport anisotropy of the designed structures, we define the directional effective mean free paths $l_{\mu}^{*}(E) \equiv \max _{t} D_{\mu}(E, t) /\left[2 v_{\mu}(E)\right]$. We note that while we find a significant renormalization of the Fermi velocity for pristine superlattices, such an effect, as discussed above, is absent for the level of disorder we consider here. We thus use the pristine graphene Fermi velocity in all calculations of mean free paths.

As is evident in Fig. 4(a), the anisotropy of the structure is readily apparent in the mean free paths $l_{\mu}^{*}$, which not only differ in magnitude, but also exhibit distinct energy dependencies, with $l_{x}^{*}(E)$ almost featureless except for a small dip near zero energy. The energy dependence of $l_{y}^{*}$ is considerably richer, with a behavior similar to that seen in Fig. 3(c), albeit with features slightly smeared by the addition of long-range impurities. Our interpretation of the energy dependence of $l_{y}^{*}$ remains the same as that discussed above in relation to Fig. 3(c). The directional mean free paths in Fig. 4(a) are shown for increasing values of $N_{x}$, illustrating how this parameter serves as an effective way of tuning the anisotropy of the scattering-mediated transport. Indeed, $l_{y}^{*}$ is approximately linearly dependent on $N_{x}$ for energies near the Dirac point. We stress that even though the directional mean free paths are of similar magnitude near the Dirac point, the transport regimes remain distinctly different, namely diffusive along the easy direction while strongly localized along the hard direction. The corresponding semiclassical conductivities are shown in Fig. 4(b), illustrating how, along the easy direction, the distinct behavior of the mean free path near zero energy is counteracted by the density of states, resulting in a largely flat plateau near the Dirac point. Note that the width of the plateau decreases as $N_{x}$ is increased, while the height of the plateau increases approximately linearly with $N_{x}$. The semiclassical conductivity along the hard direction shows an entirely different energy dependence, with a slight peak near the Dirac point. We find that the zero-energy semiclassical conductivity is nearly independent of $N_{x}$, and shows an approximately quadratic dependence on $N_{x}$ at nonzero energies near the Dirac point. The horizontal dashed line in Fig. 4(b) indicates the minimum value $\sigma_{\min }^{\mathrm{SC}}=4 e^{2} / \pi h$, a value that has been demonstrated to fix the minimum semiclassical conductivity in disordered graphene [27,31].

While the ratios $\sigma_{y y}^{\mathrm{SC}} / \sigma_{x x}^{\mathrm{SC}}$ of the semiclassical conductivities shown in Fig. 4 are less than an order of magnitude, transport along the hard direction enters the Anderson localized regime, which will further maximize the resulting transport anisotropy. To illustrate this, we study the disordered $\{16,9\}$ superlattice in absence of long-range impurities. Figure 5(a) shows the conductivities $\sigma_{\mu \mu}$ as a function of sample dimensions $L_{x}$ and $L_{y}$. The location of the minimum semiclassical conductivity $\sigma_{\min }^{\mathrm{SC}}=4 e^{2} / \pi h$, indicated by the horizontal dashed line in Fig. 5(a), separates the different transport regimes. For $\sigma_{\mu \mu}>\sigma_{\min }^{\mathrm{SC}}$ the system 

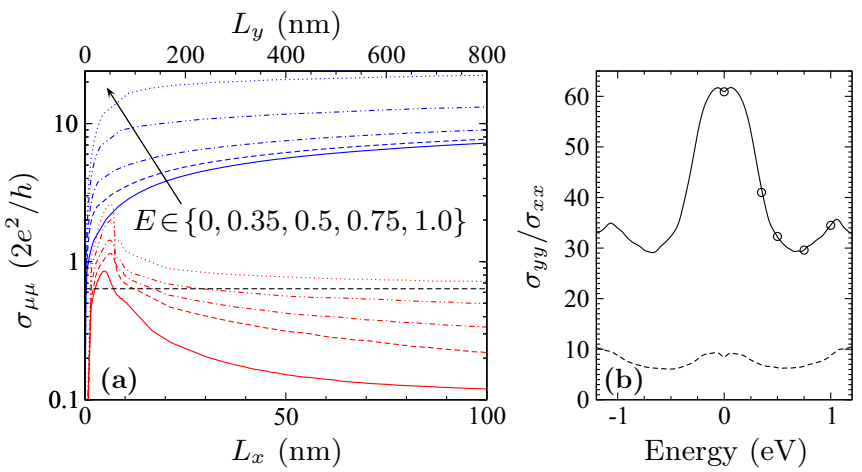

FIG. 5. (Color online) (a) Conductivity vs length for the disordered $\{16,9\}$ superlattice. Red lines show results for the "hard" direction $\sigma_{x x}$, while blue lines indicate results for the "easy" direction $\sigma_{y y}$. Note the logarithmic scale on the ordinate. The horizontal dashed line indicates the minimum value $\sigma_{\min }^{\mathrm{SC}}$ in the absence of quantum interference. (b) Ratio of conductivities calculated at the maximum lengths of the simulated time span of $20 \mathrm{ps}$. The dashed line indicates the ratio when quantum interference effects are neglected for $\sigma_{x x}$. Circles indicate the energies corresponding to the results in panel a.

remains metallic and the conductivity remains finite down to zero temperature, whereas the length-dependent decay of $\sigma_{\mu \mu}$ below $\sigma_{\min }^{\mathrm{SC}}$ is evidence of the dominant role played by quantum interferences, which successively drive the electronic state from weak localization to the strong (Anderson) localization regime.

Note that outside the initial transition regime, conductivity along the easy direction becomes more than an order of magnitude larger than along the hard direction within our accessible computational time (or length) scales. This trend is illustrated in Fig. 5(b), where the ratio $\sigma_{y y}\left(L_{y, \max }\right) / \sigma_{x x}\left(L_{x, \max }\right)$ is shown. The strongest anisotropy is seen near the Dirac point, where scattering due to edge states around the holes is most pronounced. As we further upscale sample size, the transport anisotropy $\sigma_{y y}\left(L_{y}\right) / \sigma_{x x}\left(L_{x}\right)$ will continuously increase. A crossover to very strong anisotropy will take place once the sample size along the hard direction reaches the directional localization length $\xi_{x}$. This length scale can be estimated using $\xi_{x}(E)=l_{x}^{*}(E) \exp \left[\pi h \sigma_{x x}^{\mathrm{SC}}(E) /\left(2 e^{2}\right)\right]$ [3]. For the $\{16,9\}$ superlattice in the absence of long-range impurities, the localization length along the hard direction is of the order of $10 \mathrm{~nm}$, while the localization length along the easy direction is much larger, of order of several thousands of nanometers.

In an experiment, our results suggest that depending on the measurement geometry, the low temperature conductivity could manifest a variable range hopping in the hard direction (similar to the case of damaged graphene [32]) whereas metallic behavior will be measured in the perpendicular direction. To date such strong conductivity anisotropy has never been reported in metallic materials, or disordered graphene. By applying an external magnetic field, one may further tailor the anisotropy in the semiclassical regime [33], while the modulation of magnetotransport features at low fields and the quantum Hall effect at high fields both deserve further consideration.

In conclusion, we have shown that disorder, while destroying anisotropic velocity renormalization in perfect graphene superlattices, can result in large anisotropic conductivities by inducing strong localization along the hard direction of transport. These results suggest that disorder could serve as an inherent tool in the field of nanostructured graphene, giving rise to different transport properties of massless Dirac fermions.

The work by J.G.P. is financially supported by the Danish Council for Independent Research, FTP Grants No. 11105204 and No. 11-120941. S.R. and A.W.C. acknowledge the Spanish Ministry of Economy and Competitiveness for national project funding (MAT2012-33911) and SAMSUNG for support within the Global Innovation Program. The research leading to these results has received funding from the European Union Seventh Framework Programme under Grant No. 604391 Graphene Flagship. We thank Antti-Pekka Jauho for helpful comments on the manuscript.
[1] M. I. Katsnelson, K. S. Novoselov, and A. K. Geim, Nat. Phys. 2, 620 (2006).

[2] E. McCann, K. Kechedzhi, V. I. Falko, H. Suzuura, T. Ando, and B. L. Altshuler, Phys. Rev. Lett. 97, 146805 (2006); F. V. Tikhonenko, A. A. Kozikov, A. K. Savchenko, and R. V. Gorbachev, ibid. 103, 226801 (2009); P. M. Ostrovsky, I. V. Gornyi, and A. D. Mirlin, Phys. Rev. B 74, 235443 (2006); K. Nomura and A. H. MacDonald, Phys. Rev. Lett. 98, 076602 (2007); P. M. Ostrovsky, M. Titov, S. Bera, I.V. Gornyi, and A. D. Mirlin, ibid. 105, 266803 (2010).

[3] P. A. Lee and T. V. Ramakrishnan, Rev. Mod. Phys. 57, 287 (1985).

[4] F. Evers and A. D. Mirlin, Rev. Mod. Phys. 80, 1355 (2008).

[5] K. S. Novoselov, V. I. Falko, L. Colombo, P. R. Gellert, M. G. Schwab, and K. Kim, Nature (London) 490, 192 (2012).

[6] D. A. Areshkin and C. T. White, Nano Lett. 7, 3253 (2007).
[7] J. T. Kim and S.-Y. Choi, Opt. Express 19, 24557 (2011).

[8] H. Yan, X. Li, B. Chandra, G. Tulevski, Y. Wu, M. Freitag, W. Zhu, P. Avouris, and F. Xia, Nat. Nanotechnol. 7, 330 (2012).

[9] M. Yakes, D. Gunlicke, J. L. Tedesco, P. M. Campbell, R. L. Myers-Ward, C. R. Eddy, P. E. Kurt Gaskill, D. Sheehan, and A. R. Laracuente, Nano Lett. 5, 1559 (2010).

[10] G.-X. Ni, Y. Zheng, S. Bae, H. R. Kim, A. Pachoud, Y. S. Kim, C.-L. Tan, D. Im, J.-H. Ahn, B. H. Hong, and B. Özyilmaz, ACS Nano 6, 1158 (2012).

[11] J. P. Robinson, H. Schomerus, L. Oroszlany, and V. I. Falko, Phys. Rev. Lett. 101, 196803 (2008); T. O. Wehling, S. Yuan, A. I. Lichtenstein, A. K. Geim, and M. I. Katsnelson, ibid. 105, 056802 (2010).

[12] C.-H. Park, L. Yang, Y.-W. Son, M. V. Cohen, and S. G. Louie, Nat. Phys. 4, 213 (2008). 
[13] T. G. Pedersen, C. Flindt, J. Pedersen, N. A. Mortensen, A.-P. Jauho, and K. Pedersen, Phys. Rev. Lett 100, 136804 (2008); J. A. Fürst, J. G. Pedersen, C. Flindt, N. A. Mortensen, M. Brandbyge, T. G. Pedersen, and A.-P. Jauho, New. J. Phys. 11, 095020 (2009).

[14] A. J. M. Giesbers, E. C. Peters, M. Burghard, and K. Kern, Phys. Rev. B 86, 045445 (2012).

[15] J. Eroms and D. Weiss, New J. Phys. 11, 095021 (2009).

[16] S. Yuan, R. Roldan, A.-P. Jauho, and M. I. Katsnelson, Phys. Rev. B 87, 085430 (2013).

[17] P. D. Garcia, R. Sapienza, C. Toninelli, C. Lopez, and D. S. Wiersma, Phys. Rev. A 84, 023813 (2011).

[18] T. Gunst, T. Markussen, A.-P. Jauho, and M. Brandbyge, Phys. Rev. B 84, 155449 (2011).

[19] S. Roche and D. Mayou, Phys. Rev. Lett. 79, 2518 (1997).

[20] A. Lherbier, X. Blase, Y.-M. Niquet, F. Triozon, and S. Roche, Phys. Rev. Lett. 101, 036808 (2008).

[21] H. Ishii, F. Triozon, N. Kobayashi, K. Hirose, and S. Roche, C. R. Physique 10, 283 (2009).

[22] L. E. F. Foa Torres, S. Roche, and J. C. Charlier, Introduction to Graphene-Based Nanomaterials: From Electronic Structure to Quantum Transport (Cambridge University Press, Cambridge, 2014).

[23] R. Haydock, Comput. Phys. Commun 20, 11 (1980).
[24] J. Bai, X. Zhong, S. Jiang, Y. Huang, and X. Duan, Nat. Nanotechnol. 5, 190 (2010); I. Jung, H. Y. Jang, and S. Park, Appl. Phys. Lett. 103, 023105 (2013); J. Liu, H. Cai, X. Yu, K. Zhang, X. Li, J. Li, N. Pan, Q. Shi, Y. Luo, and X. Wang, J. Phys. Chem. C 116, 15741 (2012).

[25] T. M. Radchenko, A. A. Shylau, and I. V. Zozoulenko, Phys. Rev. B 86, 035418 (2012).

[26] M. Vanević, V. M. Stojanović, and M. Kindermann, Phys. Rev. B 80, 045410 (2009).

[27] S. Roche, N. Leconte, F. Ortmann, A. Lherbier, and D. Soriano, Sol. Stat. Commun. 152, 1404 (2012).

[28] K. Nomura and A. H. MacDonald, Phys. Rev. Lett. 96, 256602 (2006).

[29] Y. Y. Zhang, J. Hu, B. A. Bernevig, X. R. Wang, X. C. Xie, and W. M. Liu, Phys. Rev. Lett. 102, 106401 (2009).

[30] F. Ortmann, A. Cresti, G. Montambaux, and S. Roche, Europhys. Lett. 94, 47006 (2011).

[31] A. Lherbier, Simon M.-M. Dubois, X. Declerck, S. Roche, Y.-M. Niquet, and J.-C. Charlier, Phys. Rev. Lett. 106, 046803 (2011).

[32] J. Moser, H. Tao, S. Roche, F. Alsina, C. M. Sotomayor Torres, and A. Bachtold, Phys. Rev. B 81, 205445 (2010).

[33] A. Barelli, J. Bellissard, and F. Claro, Phys. Rev. Lett. 83, 5082 (1999). 